\title{
Exploring the Differences in Spatial Resolution between Auger and EDS Elemental Mapping
}

\author{
Casey R. Thurber ${ }^{1}$ \\ 1. Honeywell, Kansas City, MO, USA.
}

With the expansion in the area of nanotechnology, the need for instruments with high spatial resolution becomes critical to the advancement of the analysis of nano-scale regions. Two different techniques used for elemental mapping are energy dispersive X-ray spectroscopy (EDS) and scanning Auger electron microscopy (SAM). SAM has shown excellent results compared to EDS when chemically mapping nanoscale features due to a superiority in lateral spatial resolution. Examples will be highlighted from in-situ analysis to compare the differences in spatial resolution for elemental mapping between EDS and SAM techniques.

Auger electron spectroscopy (AES) is a technique that analyzes the surface of a material to determine the elemental composition. In AES, the surface of a sample is analyzed with an electron beam usually in the range of 3-25 keV, which induces core level electrons to be ejected from the sample [1]. The resulting electrons, known as Auger electrons, are of low energy which only allows the electrons close to the surface $(\sim 1-10 \mathrm{~nm})$ to escape for detection resulting in a "true" surface analysis technique. The electron beam can be probed across the surface of the sample to create elemental maps within a given region of the sample, which is known as SAM. The spatial resolution of SAM elemental images has shown to be in the range of $6-8 \mathrm{~nm}[2]$.

EDS is a technique used as a routine analysis to analyze the elemental and structural parts of specimens. The technique works by flooding the surface of a solid sample with an electron beam (typically 3-40 keV) which produces characteristic X-rays that are detected by a silicon drift detector. The resulting X-rays are much higher in energy than the electrons produced in AES and result in a $\sim 1-3 \mu \mathrm{m}$ depth of analysis, which is usually considered more of a "bulk" analysis compared to AES. Also, the spatial resolution for EDS elemental images has shown to be in the range of $\sim 1 \mu \mathrm{m}$, which is significantly higher than SAM [3].

A PHI 710 Auger spectrometer coupled with a Bruker XFlash 6|10 EDS detector was used for this study. Figure 1 shows the analysis of the edge chemistry of a Ni-Cr layered standard (Geller Microanalytical NiCr-3 depth profiling standard) after being sputtered with $3 \mathrm{keV} \mathrm{Ar+} \mathrm{using} \mathrm{Zalar} \mathrm{rotation.} \mathrm{Parts} \mathrm{A} \mathrm{and}$ $B$ represent the SEM image and area chemically mapped using SAM with a $5 \mathrm{keV}$ electron beam. The images representing parts $\mathrm{C}$ and $\mathrm{D}$ show the SEM image and chemically mapped region using the EDS with a $10 \mathrm{keV}$ electron beam. After comparison of the SAM chemical map and the EDS chemical map, the SAM map shows excellent high resolution lines delineating the $\mathrm{Ni} / \mathrm{Cr}$ layers in distinction with the $\mathrm{Si}$ substrate. The EDS map displays a lower resolution image and has difficulty distinguishing the last $\mathrm{Cr}$ and Ni layer from the Si substrate, due to depth penetration.

In conclusion, SAM has shown superior spatial resolution and surface depth analysis when compared to EDS for chemically mapping nano-scale features. In the coming years, SAM will continue play a vital role in the development and advancement in the area of nanotechnology. 


\section{References:}

[1] D Briggs and JT Grant, Surface Analysis by Auger and X-Ray Photoelectron Spectroscopy, (IM Publications and Surface Spectra Limited, UK) p. 57-58.

[2] SN Raman et al, Microsc. Microanal. 16 S2 (2010) p. 354-355.

[3] JJ Friel et al, X-ray and Image Analysis in Electron Microscopy, (Bruker Nano GmbH, Berlin) p. 10$11,24$.
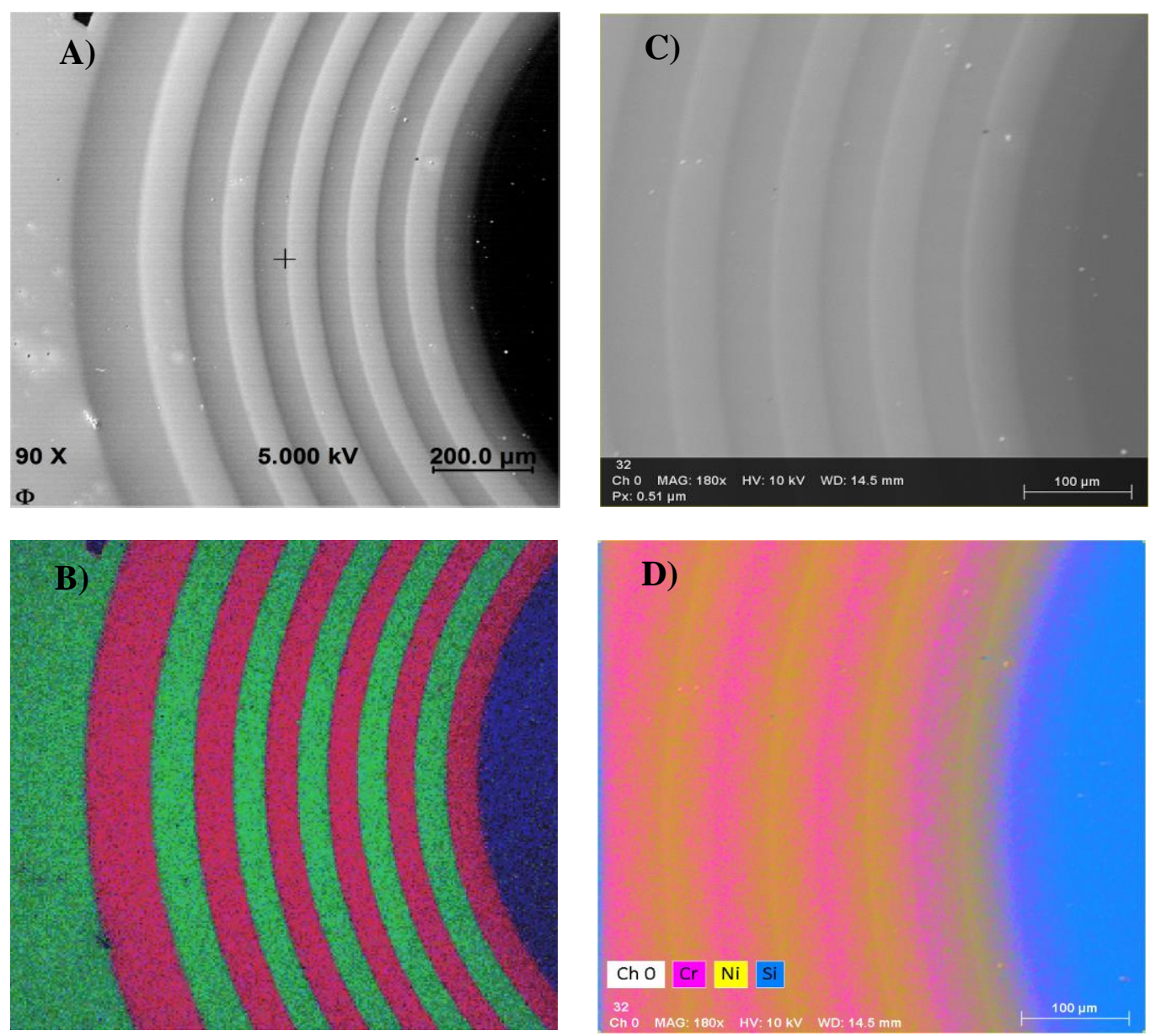

Figure 1: Ni-Cr layered standard after sputtering with 3kV Ar+ where A) represents an SEM image of a chemically mapped area B) using Auger electron spectroscopy $($ Red $=$ Chromium, Green $=$ Nickel, and Blue $=$ Silicon) and C) represents an SEM image of a chemically mapped area D) using energydispersive x-ray spectroscopy. 